\title{
Gobiernos Locales en Bolivia
}

\author{
Local Governments in Bolivia
}

\author{
Álvaro Israel Deuer Cenzano \\ deueral@gmail.com \\ https://orcid.org/0000-0001-8876-4483
}

Universidad Católica Boliviana, La Paz-Bolivia

\section{Palabras clave}

ABSTRACT

Keywords
El objetivo de la investigación fue analizar la situación de los Gobiernos Locales en lo que se refiere a los Gobiernos Autónomos Municipales (GAM) en Bolivia, especialmente de aquellos que cuentan con escasa población y recursos económicos, como línea de base para explorar soluciones de política pública que permitan mejorar las condiciones de vida de su población. Para esto, primero se describió el marco normativo de la creación y fusión de unidades territoriales (UT); a continuación, se muestra la evolución de la división territorial municipal en Bolivia (1995 - 2021); finalmente, se analizazon sus principales fuentes de financiamiento, el destino de su gasto y los problemas asociados con estas variables. Como resultado, se encontró que, en el ámbito económico, los GAM de menos de 5000 habitantes tienen capacidades institucionales deficientes, a causa de: escasa población, falta de desarrollo de capacidades fiscales y dificultad para ejecutar recursos. Esto pone en cuestión su habilidad para cumplir con las garantías, mandatos y competencias asignadas a este nivel de gobierno en la Constitución Política de Estado (CPE), en áreas prioritarias para el desarrollo del Estado como son los sectores de salud y educación.

Amalgamación; Gobiernos locales; Capacidades institucionales; Pereza fiscal
The research' objective was to analyze the situation of Local Governments in Bolivia on its Municipal Layer (GAM), especially those that have scarce population and economic resources, as a baseline to explore public policy solutions to improve the living conditions of their inhabitants. For this purpose, first, the regulatory framework for its creation and merge was reviewed; Next, the evolution of their territorial division in Bolivia is shown (1995 - 2021); Finally, it analyzed its main revenues, expenditures, and the problems associated with these variables. As a result, in the economic sphere, it was found that GAM of less than 5,000 inhabitants shows weak institutional capacities, has minor sources of own income, and deploys low rates of budget execution. That threatens their ability to comply with the guarantees, mandates, and competences assigned to them by the Bolivian Constitution (CPE) in priority areas for the development of the State such as the health and education sectors, is questioned.

Amalgamation; Local governments; Institutional capacities; Fiscal laziness 
INTRODUCCIÓN

Durante la segunda mitad del siglo XX hubo una proliferación de reformas territoriales que surgieron del desarrollo de técnicas para la determinacióndeltamañoóptimo(anivelterritorial y poblacional) que debían tener los gobiernos locales para la prestación eficiente y eficaz de las mayores y más amplias responsabilidades que les habían sido transferidas como fruto de los procesos de descentralización y así garantizar la calidad de los servicios públicos asociados a éstas, dando lugar a nuevas entidades territoriales a través de procesos de fusión (amalgamación) normalmente organizados de arriba hacia abajo, bajo la premisa de que con Unidades Territoriales (UTs) más grandes se alcanzarían mayores impactos a menores costos unitarios.

El surgimiento de efectos de economía de escala a la par del incremento del tamaño de las unidades territoriales de los gobiernos locales, conocido como "eficiencia productiva" (Taveres, 2018), tiene su origen en las teorías de optimización económica que igualaban los beneficios marginales a los costos marginales. Equiparando la producción de servicios públicos con la población, se esperaba que los costos de producción por unidad decrecieran a medida que cierta cantidad del producto fuera producida y luego se incrementara más allá de cierto punto, en función de las propiedades que presenta una curva de costo marginal con forma de $U$ (Hellsing, 2012). Sin embargo, la aplicación de esta teoría mostró que las economías de escala no son uniformes en la diferente gama de servicios que son provistos por los gobiernos locales.

En el caso boliviano, las entidades locales revelan tener -mayoritariamente - una base poblacional disminuida. Si se define como GAM pequeño a todo aquel que registre un máximo de 15.000 habitantes. Cerca de dos tercios de los GAM $(64,29 \%)$ se encuentran en esta situación debido a que 216 GAM, de los 336 que existen en la actualidad, no rebasan el techo poblacional considerado (Instituto Nacional de Estadística, 2021).

Este factor asociado a las debilidades financieras plantea limitaciones para el ejercicio de las competencias y el cumplimiento de las tareas del desarrollo y lucha contra la pobreza. La mayor parte de los GAM pequeños se encuentran en regiones alejadas de los grandes centros urbanos y adolecen por ello, de importantes déficits en infraestructura productiva y social, todo lo cual tiende a disminuir su capacidad institucional y financiera.

La elevada heterogeneidad poblacional es percibida como un obstáculo para la descentralización, por lo que surge la necesidad de impulsar planes de ordenamiento territorial (Böhrt, 2011).

En este contexto, el objetivo de este estudio fue mostrar la situación de los GAM en Bolivia, especialmente de aquellos que cuentan con escasa población (menos de 5.000 habitantes) y recursos económicos que sirva como línea de base para explorar soluciones de política pública que permitan hacerlos viables y así incidir en la mejora de las condiciones de vida de su población.

\section{METODOLOGÍA}

Este estudio tuvo alcance descriptivo desarrollado bajo un enfoque positivista, con el objetivo de mostrar la situación de los GAM en Bolivia, especialmente de aquellos que cuentan con escasa población y recursos económicos que sirva como línea de base para explorar soluciones de política pública que permitan hacerlos viables y así incidir en la mejora de las condiciones de vida de su población.

Las principales fuentes de información consultadas fueron: Censo Nacional de Población y Vivienda 2012 (CNPV); Base de Datos de Entidades Territoriales del Servicio Estatal de Autonomías (SEA); Ministerio de Economía y Finanzas Públicas 
(MEFP) y; Deuer \& Aparicio (2018). A continuación, se detalla los criterios de selección de las fuentes de información y algunas otras consideraciones importantes.

El indicador de necesidades básicas insatisfechas (NBI) se descompone en componentes relacionados con la vivienda (materiales de vivienda, espacios) y servicios e insumos energéticos (agua y saneamiento ambiental e insumos energéticos). En Bolivia el indicador de las NBI se calcula con los datos censales que siguiendo protocolos internacionales se realiza aproximadamente cada 10 años. Si bien las Encuestas de Hogares contienen un módulo de vivienda, al constituirse ésta en una estimación muestral, su factor de expansión es insuficiente para generalizar sus resultados a todo el universo. Por este motivo, los datos de las $\mathrm{NBI}$ de este estudio corresponden a los valores calculados por el Instituto Nacional de Estadística (INE) en base al Censo Nacional de Población y Vivienda 2012 (CNPV).

La información de población se recopilo de las estimaciones realizadas por el INE para 2021 en base a los datos del CNPV 2012.

La información del Presupuesto de Ingresos y de Gastos fue extraída de la Base de Datos de Entidades Territoriales del SEA. En todos los casos se utilizó los presupuestos devengados con las siguientes salvedades: (1) Para los datos faltantes en el presupuesto de ingresos para los GAM con código municipal 1906 al 1915 (todos correspondientes al Departamento de Pando), se utilizaron los datos del presupuestados publicados en la página WEB del MEFP; (2) Para los 13 GAM cuyos datos de presupuesto devengando en la base eran 0 o menores a $200.000 \mathrm{Bs}$, por un criterio de comparabilidad, se utilizaron los datos del presupuesto de ingresos publicados en la página WEB del MEFP.

En el caso del sector de educación se verifico la existencia de datos actualizados al 2020 con desagregación municipal con la Unidad de Análisis de Política Económica del Ministerio de Planificación del Desarrollo (MPD) y en la página Web del Ministerio de Educación. Al constar en ambas fuentes que lo datos actualizados del Sistema de Estadísticas e Indicadores Educativos solo presentaban una desagregación Departamental, se tomó la decisión de utilizar los datos de Deuer \& Aparicio (2018). En el caso del sector de salud los datos fueron recogido de Deuer \& Aparicio (2018), cuya base es el año 2016.

\section{RESULTADOS}

Se analizó la organización territorial del Estado Plurinacional de Bolivia con énfasis en la situación de los GAM, desde lo establecido en la CPE de 1967 hasta la actualidad. Éste contempla un examen del tamaño de los GAM (superficie), localización, población, grado de pobreza, acceso a servicios de educación, salud, fuentes de financiamiento y el impacto de estas variables en sus capacidades institucionales para prestar servicios básicos a la población y cumplir sus fines.

\section{Marco normativo}

Según el artículo 14 de la Ley No. 031 (Ley marco de autonomías y descentralización, 2010), la organización territorial tiene como finalidad fortalecer la cohesión territorial, estableciendo un sistema de organización del territorio que configure unidades funcionales y espacialmente integradas de forma armónica y equilibrada (Ley marco de autonomías y descentralización, 2010). El territorio del Estado Plurinacional de Bolivia se organiza para un mejor ejercicio del gobierno y la administración pública en UT que pueden tomar la forma de departamentos, provincias, municipios y Territorios Indígena Originario Campesinos (TIOC). En la actualidad Bolivia se subdivide en 463 UT según se presenta en la siguiente Tabla. 
Tabla 1. Organización territorial de Bolivia a junio 2021.

\begin{tabular}{cccccc}
\hline Departamento & Provincias & GAM & AIOC & GAR & TOTAL - UT \\
\hline Chuquisaca & 10 & 29 & & & 39 \\
La Paz & 20 & 87 & & 107 \\
Cochabamba & 16 & 47 & 1 & 64 \\
Oruro & 16 & 33 & $2^{(1)}$ & & 51 \\
Potosí & 16 & $41^{(3)}$ & & 1 & 57 \\
Tarija & 6 & 11 & & & 18 \\
Santa Cruz & 15 & 54 & $2^{(2)}$ & & 71 \\
Beni & 8 & 19 & & & 27 \\
Pando & 5 & 15 & & & 20 \\
Total 9 & 112 & 336 & 5 & 1 & 463 \\
\hline
\end{tabular}

Fuente: Elaborado con datos del SEA (Servicio Estatal de Autonomías, 2021), MEFP (Ministerio de Economía y Finanzas Públicas, 2021), INE (Instituto Nacional de Estadística, 2021)

Según datos presentados en la Tabla 1, corresponde aclarar: (1) No se contempla a Salinas de Garci Mendoza del departamento de Oruro que se convirtió de GAM a GAIOC (Gobierno Autónomo Indígena Originario Campesino); (2) en el departamento de Potosí se considera al GAM de San Pedro de Macha; (3) en el departamento de Santa Cruz se toma en cuenta a Gutiérrez Keremba lyambae que se convirtió de GAM a GAIOC. Por otra parte, se debe notar en las Directrices y Clasificador Presupuestario 2021 (2020), el GAM de San Pedro de Macha y los GAIOC de Salinas de Garcí Mendoza y de Gutiérrez no tienen asignados códigos institucionales; sin embargo, sus autoridades fueron electas para el período 2021 - 2026, en las elecciones del 7 de marzo de 2021 en el caso del GAM de San Pedro de Macha, y de acuerdo con sus usos y costumbres en elecciones supervisada por el Tribunal Supremo Electoral (TSE) en el caso de Salinas de Garcí Mendoza y de Gutiérrez. Asimismo, debe considerarse que las Directrices y Clasificador Presupuestario 2021 (2020) contempla códigos institucionales solo para los GAIOC del Territorio de Raqaypampa, la Nación Originaria Uru Chipaya, y de Charagua lyambae.

Nótese que la creación y modificación de UT anteriormente era regulada por la Ley No. 2150 (Ley de Unidades Político Administrativas, 2000). Sin embargo, la Ley No. 031 (Ley marco de autonomías y descentralización, 2010) dispuso la suspensión temporal de la admisión de nuevas solicitudes de creación, supresión y/o anexión de UT, hasta que se dicte una nueva normativa.

Actualmente, la Entidad Territorial Autónoma (ETA) es la institucionalidad que administra y gobierna en la jurisdicción de una UT. En Bolivia todas las UT, con excepción de la provincia, conforman gobierno. La creación, modificación y delimitación de nuevas UT debe sujetarse a lo establecido en Ley especial que regule las condiciones y procedimientos para tal efecto, debiendo cada caso ser aprobado por Ley de la Asamblea Legislativa Plurinacional (ALP) (Constitución Política del Estado, 2009). En la actualidad, la delimitación de UT se realiza con base en la regulación establecida por la Ley No. 339 (Ley de delimitación de Unidades Territoriales

ISSN: 2788 - 6557| ISNN-L: 2788 - 6557
Volumen 3 | No. 2| Julio - diciembre 2021
www.revistapanel.org


, 2013) y el DS No. 1560 (Reglamento de la Ley $N^{\circ}$ 339, de 31 de enero de 2013, de Delimitación de Unidades Territoriales, 2013). La delimitación de UT interdepartamentales e intradepartamentales se realiza a través de un procedimiento de conciliación administrativa en el marco de la voluntad democrática de sus habitantes mediante referéndum.

\section{Diagnóstico de la situación de los Gobiernos Locales en Bolivia}

En cuanto al régimen municipal, la CPE de 1967 (Constitución política del Estado, 1967), garantizaba la autonomía de los gobiernos comunales (Molina, 1997). Establecía que en las capitales de departamento haya Consejos Municipales y un alcalde rentado. En cambio, para provincias, sus secciones y los puertos, mandaba la constitución de juntas municipales. Esta denominación diferenciada no era gratuita. Dicho cuerpo normativo también autorizaba a los Consejos de las capitales de departamento y a sus alcaldes a ejercer supervigilancia y control sobre las juntas y alcaldes provinciales. Por otro lado, este cuerpo normativo también indicaba que mediante ley se delimitaría la jurisdicción territorial de cada municipio. Es decir, el parlamento debería definir caso por caso (Molina, 1997).

La Ley No. 686 (Ley Orgánica de Municipalidades, 1985), promulgada en enero de 1985 , entendía las necesidades del municipio casi exclusivamente como las propias del municipio urbano: desarrollo arquitectónico, vialidad y ornato público, etc. Además, mantenía la definición de la jurisdicción de cada municipio como una atribución del Parlamento nacional, absteniéndose de fijar, ella misma, un criterio general (Molina, 1997).

Como se puede observar, la CPE y la Ley No. 686 (Ley Orgánica de Municipalidades, 1985), consideraban que cualquier ciudad y pueblo, sin importar su tamaño, podía constituirse en municipio. Sin embargo, en los hechos, éste tenía vigencia sólo en los principales centros del país. En las localidades menores no funcionaba, o porque sus habitantes no habían gestionado su creación ante el Parlamento, o porque existiendo legalmente, no tenían utilidad real ni recursos suficientes (Molina, 1997).

La CPE (Constitución Política del Estado, 1967), con reformas parciales acordadas mediante Ley No. 1473 (1993) y Ley No 1585 (Reforma parcial de la CPE de 1967, 1994), si bien suprime la distinción entre Consejos y juntas vecinales (igualdad jurídica), mantiene que el territorio municipal sea definido por Ley. Es la Ley No. 1551 (Ley de Participación Popular, 1994).

Si bien lo recomendable hubiese sido realizar estudios previos para la delimitación municipal, se consideró que en general lo más práctico era transformar en municipios a las secciones de provincia, decisión que a criterio de Víctor Hugo Cárdenas (ex vicepresidente de Bolivia entre 1993 -1997) se tomó sin seguir ningún criterio territorial, social, cultural o científico, quedando pendiente el debate sobre el reordenamiento territorial (Finot, 2016). El efecto de este pragmatismo fue el nacimiento de 312 municipios con población escasa y dispersa, con limitaciones de tipo económico, institucional (recursos humanos, físicos y financieros) y territorial (problemas limítrofes) que condicionaron de origen, la posibilidad de administrar eficientemente un municipio y de prestar servicios oportunamente para lograr el bienestar de la población (Ameller, 1999).

En la Tabla 2 se presenta la distribución por departamento de los municipios con menos de 5000 habitantes. 
Tabla 2. GAM con menos de 5000 habitantes 2021 Bolivia.

\begin{tabular}{ccccc}
\hline Departamento & Total GAM & $\begin{array}{c}\text { Municipios } \\
<\mathbf{5 0 0 0} \text { Hab 2021p }\end{array}$ & \% Dept. & \% Bolivia \\
\hline Chuquisaca & 29 & 5 & $17,2 \%$ & $6,8 \%$ \\
La Paz & 87 & 14 & $16,1 \%$ & $18,9 \%$ \\
Cochabamba & 47 & 8 & $17,0 \%$ & $10,8 \%$ \\
Oruro & 33 & 20 & $60,6 \%$ & $27,0 \%$ \\
Potosí & 41 & 9 & $22,0 \%$ & $12,2 \%$ \\
Tarija & 11 & 0 & $0,0 \%$ & $0,0 \%$ \\
Santa Cruz & 54 & 5 & $9,3 \%$ & $6,8 \%$ \\
Beni & 19 & 5 & $26,3 \%$ & $6,8 \%$ \\
Pando & 15 & 8 & $53,3 \%$ & $10,8 \%$ \\
Total 9 & 336 & 74 & & $100 \%$ \\
\hline
\end{tabular}

Fuente: Elaborado con datos del INE (Instituto Nacional de Estadística, 2021).

Según se presenta en la Tabla 2 , de acuerdo con la categorización por cantidad de habitantes, en la actualidad 74 GAM en Bolivia cuentan con menos de 5000 habitantes, estando el $58 \%$ de éstos localizados en el occidente de Bolivia en los departamentos de Oruro, La Paz y Potosí (27\%, $18,9 \%, 12,2 \%$ respectivamente). A su vez, $60,6 \%$ de los GAM del departamento de Oruro y $53,3 \%$ de los GAM del departamento de Pando se encuentran en esta franja. Por otro lado, se debe destacar que el $100 \%$ de los GAM del Departamento de Tarija cuentan con 5000 habitantes o más.

Por otro lado, como se muestra en la Tabla 3, en el periodo 1995 - 2021, el número de GAM creció en un $7,7 \%$, alcanzando un techo de 339 en 2017 (Directrices y Clasificadores
Presupuestarios, 2016), siendo Santa Cruz y La Paz los departamentos que concentraron la mayor cantidad de creaciones, pasando de tener 48 a 56 y 75 a 87 GAM, lo que representa un crecimiento del $16 \%$ y del $16,67 \%$ respectivamente con relación a las UT que tenían inicialmente y explicaban el $74,1 \%$ de las nuevas UT locales a nivel nacional. Posteriormente este número disminuyó hasta los 336 GAM que existen en la actualidad. Esto por la conversión de diversos GAM en GAIOC (Charagua, Chipaya, Salinas de Garcí Mendoza) y la creación del GAM de San Pedro de Macha. Se espera que este número disminuya debido que a que diversos GAM se encuentran en proceso de conversión a GAIOC. 
Tabla 3. Evolución de la división administrativa municipal (1995 - 2021).

\begin{tabular}{|c|c|c|c|c|c|c|c|c|c|c|c|c|c|c|}
\hline Departamento & 1995 & $\begin{array}{l}\text { GAM } \\
<5000\end{array}$ & $\%$ & 2017 & $\begin{array}{r}\text { GAM } \\
<5000\end{array}$ & $\%$ & $\begin{array}{c}\text { Nuevos GAM } \\
1995-2017\end{array}$ & 2021 & $\begin{array}{c}\text { GAM } \\
<5000\end{array}$ & $\%$ & $\begin{array}{c}\text { Nuevos GAM } \\
2017-2021\end{array}$ & $\begin{array}{c}\Delta \text { GAM } \\
1995-2021 \\
\text { Dept. }\end{array}$ & $\begin{array}{c}\text { Nuevos } \\
\text { GAM } \\
1995-2021\end{array}$ & $\begin{array}{c}\Delta \text { GAM } \\
\text { 1995-2021 } \\
\text { Bolivia }\end{array}$ \\
\hline Chuquisaca & 28 & 4 & $14,3 \%$ & 29 & 4 & $13,8 \% 6$ & 1 & 29 & 5 & $17,2 \%$ & & $3,6 \%$ & 1 & $3,6 \%$ \\
\hline La Paz & 75 & 20 & $26,7 \%$ & 87 & 12 & 41,456 & 12 & 87 & 14 & $16,1 \%$ & & $16,0 \%$ & 12 & $42,9 \%$ \\
\hline Cochabamaba & 44 & 9 & $20,5 \%$ & 47 & 4 & $13,8 \% 6$ & 3 & 47 & 8 & $17,0 \%$ & & $6,8 \%$ & 3 & $10,7 \%$ \\
\hline Oruro & 34 & 22 & $64,7 \%$ & 35 & 16 & $55,2 \%$ & 1 & 33 & 20 & $60,6 \%$ & -2 & $-2,9 \%$ & 1 & $3,6 \%$ \\
\hline Potosi & 38 & 9 & $23,7 \%$ & 40 & 8 & $27,6 \% 6$ & 2 & 41 & 9 & $22,0 \% 6$ & 1 & 7,996 & 3 & $10,7 \%$ \\
\hline Tarija & 11 & 0 & 0,056 & 11 & 0 & $0,0 \%$ & 0 & 11 & 0 & $0,0 \%$ & & $0,0 \%$ & 0 & $0,0 \%$ \\
\hline Santa Cruz & 48 & 11 & $22,9 \%$ & 56 & 5 & $17,2 \%$ & 8 & 54 & 5 & $9,3 \%$ & -2 & $12,5 \%$ & 8 & $28,6 \%$ \\
\hline Beni & 19 & 7 & $36,8 \%$ & 19 & 3 & $10,3 \% 6$ & 0 & 19 & 5 & $26,3 \%$ & & $0,0 \%$ & 0 & $0,0 \%$ \\
\hline Pando & 15 & 14 & $93,3 \%$ & 15 & 8 & $27,6 \% 6$ & 0 & 15 & 8 & $53,3 \%$ & & $0,0 \%$ & 0 & $0,0 \%$ \\
\hline TOTAL & 312 & 96 & & 339 & 60 & & 27 & 336 & 74 & & 1 & & 28 & $100,0 \%$ \\
\hline
\end{tabular}

Fuente: Elaboración con datos del SEA (Servicio Estatal de Autonomías, 2021), MEFP (Ministerio de Economía y Finanzas Públicas, 2021), INE (Instituto Nacional de Estadística, 2021) y Ameller (1999)

Continuando con el análisis, se puede observar que los GAM de La Rivera y de Yunguyo del Litoral, ambos localizados en el departamento de Oruro, cuentan con la menor cantidad de habitantes, apenas 383 y 459 respectivamente. Llama la atención que la suma de la población de los GAM que conforman la Provincia de Mejillones en el departamento de Oruro no supera los 5 mil habitantes. A su vez la población de los GAM que conforman la provincia de Litoral en el departamento de Oruro y Abuna en el departamento de Pando, apenas supera esta cantidad.

En cuanto a la geolocalización, la Figura 1 muestra que, los GAM de menos de 5.000 habitantes, se concentran principalmente en el norte y el sudoeste del territorio de Bolivia, encontrándose entre estos el mayor porcentaje de población pobre en Bolivia. De acuerdo con los datos del INE, en 2021 existen en Bolivia 11 GAM con menos de 1000 habitantes, 7 de los cuales se en encuentran en el departamento de Oruro. De estos, 3 (42,85\%), Yunguyo del Litoral, Carangas y Choquecota presentan unas Necesidades Básicas Insatisfechas (NBI) > al 90\%. A su vez, 15 de los 74 GAM con menos de 5.000 habitantes $(20,27 \%)$ presentan un NBI mayor al $90 \%$, lo que sugiere una relación inversa entre población y pobreza.

Figura 1. GAM con menos de 5000 habitantes (año 2021).

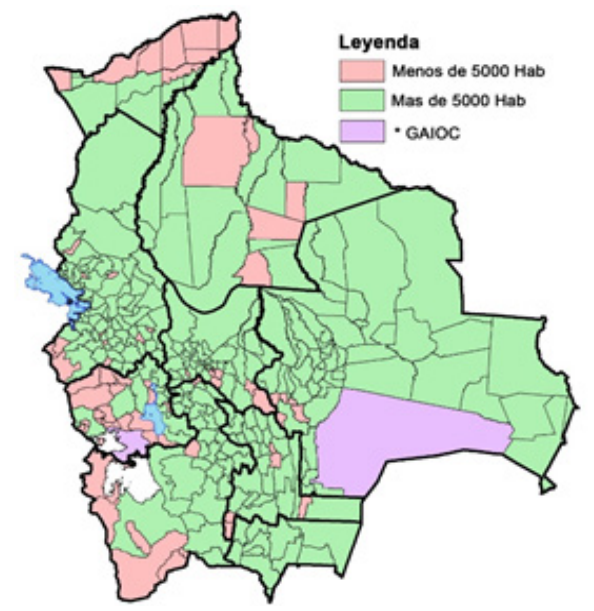

Fuente: SEA (Servicio Estatal de Autonomías, 2021) 
En cuanto a ingreso, los GAM pueden generar recursos económicos a través de su esfuerzo fiscal por ingresos de operación, venta de bienes y servicios, regalías, contribuciones a la seguridad social, tasas, derechos, multas y otros que resultan de su actividad propia, mismos que son agrupados en el rubro de recursos específicos (Directrices y Clasificadores Presupuestarios, 2020), para lo cual se requiere el desarrollo de capacidades fiscales. Analizados los datos de recursos, se observa que, de los 74 GAM con menos de 5000 habitantes, 31 (42\%) no hacen uso de esta fuente.

Figura 2. GAM Recursos devengados de los GAM de < de 5000 hab 2020.

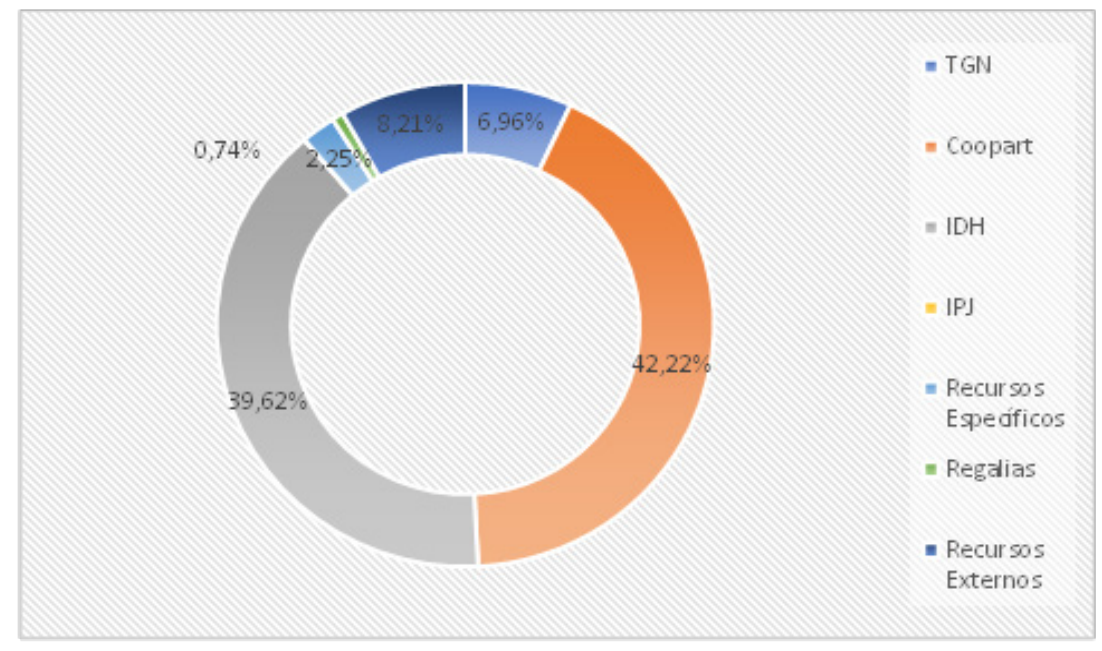

Fuente: Elaborado con datos del SEA (Servicio Estatal de Autonomías, 2021)

Con relación a los datos \% de recursos devengados presentado en la Figura 2 , se aclara: (1) la fuente TGN incluye la suma de los Organismos Financiadores 111 Tesoro General de la Nación, 114 Recursos Contravalor, 115 Donaciones HIPC II, y 117 Tesoro General de la Nación Impuesto Especial a los Hidrocarburos y sus Derivados; (2) la fuente Recursos Específicos incluye la suma de los Organismos Financiadores 210 Recursos específicos de los GAM y AICO y 230 Otros Recursos Específicos; (3) la fuente Recursos Externos incluye la suma de todos los Organismos Financiadores externos, sean estos multilaterales, bilaterales, gobiernos extranjeros u otros.

Esto incide en su alta dependencia de las transferencias del Nivel Central del Estado (NCE), especialmente por Coparticipación Tributaria e Impuesto Directo a los Hidrocarburos (IDH), que representan en promedio el $81,84 \%$ de sus recursos en 2020 , y explican el $100 \%$ de los recursos de los GAM de Charaña y Cruz de Machacamarca en los departamentos de La Paz, y Oruro respectivamente durante esta gestión, dando lugar a la aparición de pereza fiscal. Asimismo, en 34 de los 74 GAM con menos de 5000 habitantes (45\%), estas fuentes de ingreso representan conjuntamente más del $90 \%$ de sus ingresos.

Asimismo, como se muestra en el Figura 2, el acceso a recursos a través de financiamiento externo es bajo, representando $8,21 \%$ de sus recursos, existiendo 31 GAM con menos de 5000 habitantes (42\%) que no registran ingresos de esta fuente de financiamiento. Esto demuestra su limitada capacidad de apalancamiento y de gestión de recursos a través de operaciones de crédito público. Actualmente, los GAM pueden acceder 
al crédito como fuente para el financiamiento de gastos de inversión en el marco del límite máximo de endeudamiento, $20 \%$ de los ingresos corrientes recurrentes de la gestión anterior. A su vez, el valor presente de la deuda total no puede exceder el $200 \%$ de los ingresos corrientes de la gestión anterior (Normas Básicas del Sistema de Crédito Público, 1997).

En este contexto, debe destacarse la importancia que tienen la Transferencias TGN (Tesoro General de la Nación) para el financiamiento de los GAM de menos de 5 mil habitantes, especialmente aquellas registradas el Rubro 23211, Subsidios y Subvenciones del Órgano Ejecutivo. Estas hacen referencia a recursos que el NCE otorga para compensar a aquellos GAM con reducidos recursos, a través de transferencias del Fondo Nacional de Inversión Productiva y Social, Fondo Nación de Desarrollo Regional, o de Programas como Evo Cumple y otros, que pueden ser revertidos si no son utilizados para lo que se dispuso en la gestión fiscal correspondiente. A diferencia de los recursos provenientes de IDH y Coparticipación Tributaria, la asignación de Subsidios y Subvenciones del Órgano Ejecutivo es discrecional de las instituciones del NCE, por lo que existen GAM que no se benefician de esta fuente de recurso o que se benefician solo algunas gestiones.

En 2020 este tipo de transferencias representaron el $6,96 \%$ de los ingresos de los GAM con menos de 5000 habitantes, y, más del $55 \%$ de los recursos del GAM Tito Yupanqui y más del $40 \%$ de los del GAM de Puerto Siles en los Departamentos de La Paz y Beni respectivamente.

Según lo expuesto, la debilidad institucional de estas ETA, determina que en 2020 existan 19 GAM con menos de 5000 habitantes con presupuesto devengados anual menor a 1000 000 de BOB al año (equivalente a 143 678,2 \$ al tipo de cambio oficial 6,96 BOB/\$); 8 de los cuáles $(42,10 \%)$ se localizan en el departamento de Oruro. Esto quiere decir que por ejemplo los GAM de La Rivera, Yunguyo del Litoral, Huachacalla y Coipasa en el departamento de Oruro y San Antonio del Escormuco en el departamento de Potosí, disponen respectivamente de $284,93 \mathrm{BOB}(40,94 \$)$, 898,21 BOB $(129,02 \$), 819,7$ BOB $(117,68 \$), 204,94$ BOB $(29,44 \$)$ y 676,3 BOB $(97,17 \$)$ al año para atender a un ciudadano en estos GAM. Ello no solo acentúa su dependencia de las transferencias del NCE, sino que limita y determina su capacidad de acción y gestión.

Corresponde analizar la problemática expuesta por Deuer (2016):

Con los recursos públicos se debe financiar, no solo el sueldo de los funcionarios y el mantenimiento de instituciones encargadas de administrar unidades territoriales, sino el desarrollo de competencias cuya ejecución requiere de la disposición de recursos humanos con conocimientos específicos y de una inversión intensiva de recursos financieros como ser proyectos de infraestructura productiva, servicio de alumbrado público en su jurisdicción, sistemas de micro riego y construcción de caminos vecinales (Deuer, 2016, p. 28).

Por otro lado, la CPE establece que la Gestión del Sistema de Educación y Salud será ejercido de forma concurrente por el NCE y las ETA, habiendo sido distribuidas las responsabilidades referidas al sector educativo en la Ley No. 070 (Ley de la Educación Avelino Siñani - Elizardo Pérez , 2010) y las relacionadas con el sector de salud, de forma inicial por la Ley No. 031 (Ley marco de autonomías y descentralización, 2010). En este sentido es interesante analizar los datos de la ejecución de gastos de los GAM en Salud y educación, según se presenta en la Tabla 4. 
Tabla 4. Evolución de la división administrativa municipal (1995 - 2021).

\begin{tabular}{|c|c|c|c|c|c|c|c|c|c|}
\hline & \multicolumn{4}{|c|}{ Educación } & \multicolumn{3}{c|}{ Salud } & & \\
\hline GAM hab & Inicial & Devengado & $\begin{array}{c}\% \\
\text { Ejecutado }\end{array}$ & Inicial & Devengado & $\begin{array}{c}\text { \% } \\
\text { Ejecutad }\end{array}$ & $\begin{array}{c}\text { Educación + Salud } \\
\text { devengado }\end{array}$ & $\begin{array}{c}\text { Presupuesto } \\
\text { total devengado }\end{array}$ & $\begin{array}{c}\text { \% de Ejecución } \\
\text { salud + educación }\end{array}$ \\
\hline GAM > a 5000 & $8992.787 .032,00$ & $54,803.750,27$ & 6,14 & $886.425 .527,00$ & $154,418.574,56$ & 17,42 & $209.222 .324,83$ & $4.793 .804,676,25$ & 4,36 \\
\hline GAM $<=$ a 5000 & $16.277 .500,00$ & $1.217 .205,40$ & 7,48 & $18.227 .631,00$ & $2894.232,94$ & 15,88 & $4.111 .438,34$ & $82.520 .332,21$ & 4,98 \\
\hline Total & $909.064 .532,00$ & $56.020 .955,67$ & 6,16 & $904,653.158,00$ & $157.312 .807,50$ & 17,39 & $213.333 .763,17$ & $4.876 .325 .008,46$ & 4,37 \\
\hline
\end{tabular}

Fuente: Elaborado con datos del SEA (Servicio Estatal de Autonomías, 2021)

Entre las competencias asignadas, se puede observar que los GAM con población menor a 5000 habitantes consideraron entre las más importantes, aquellas relacionadas con los sectores educación y salud, asumiendo la responsabilidad de canalizar una mayor cantidad de recursos para mejorar la calidad de la infraestructura de estos servicios constituyéndose en una de las prioridades al momento de elaborar sus Programas Operativos Anuales (Ameller, 1999). Como se muestra en la Tabla 4, el porcentaje de ejecución de los gastos de educación y salud en 2020 para los GAM de menos de 5000 habitantes es de $7,48 \%$ y de $15,88 \%$. A su vez los gastos conjuntos de salud y educación representan el $4,98 \%$ de los gastos devengados por este tipo de GAM.

En el caso del sector educativo, la accesibilidad es un criterio fundamental. La distancia, las vías de comunicación, la presencia y frecuencia de medios de transporte, el costo, el tiempo que ocupa el acceso a las unidades educativas, son variables que inciden en los años de estudio que las personas en edad escolar (6 a 19 años) son capaces de alcanzar en una UT determinada (Ameller, 1999). Considerando que: de acuerdo con el Artículo 17 de la norma fundamental boliviana, toda persona tiene derecho a recibir educación en todos los niveles de manera universal, productiva, gratuita integral e intercultural, sin discriminación y; asimismo, el artículo 81.l de dicho cuerpo normativo estipula que la educación es obligatoria hasta el bachillerato (Constitución
Política del Estado, 2009); corresponde afirmar que actualmente estos derechos no están siendo suficientemente garantizado por este tipo de GAM.

La incapacidad de proveer las condiciones referidas explica que ninguno de los GAM de menos de 5000 habitantes sea capaz de garantizar la educación hasta el bachillerato, siendo el promedio de 7,5 años. Asimismo, en el 13,33\% de estos GAM (Alalay, Arampampa, Sacabamba, Curva, El Villar, Quiabaya, Moro Moro y Urmiri), la cantidad promedio de años de estudio es menor a 6 años, lo que quiere decir que en estas ETA los GAM no se puede garantizar a sus habitantes ni siquiera la finalización del ciclo primario.

En el caso del sector de salud, la asignación de personal calificado es reducida, lo que determina que el tipo de establecimientos al que pueden acceder los habitantes de los GAM de menos de 5000 habitantes sean Puestos y Centros de Salud, siendo capaces de proveer servicios médicos relacionados únicamente con la atención básica y paliativa.

Finalmente, el $23,33 \%$ de los GAM de menos de 5000 habitantes solo cuentan con un centro de atención de primer nivel, y el 19,6\% de éstos es atendido por un profesional médico con formación general, lo que demuestra que los hospitales e institutos especializados, así como el personal especializado, se concentran en las capitales de departamento. Es importante mencionar que de acuerdo con lo establecido en el Artículo 81 de la Ley No. 031 (Ley marco de autonomías y

ISSN: 2788 - 6557| ISNN-L: 2788 - 6557
Volumen 3 | No. 2| Julio - diciembre 2021
www.revistapanel.org


descentralización, 2010), es responsabilidad de los GAM administrar la infraestructura y equipamiento de los establecimientos de salud de primer y segundo nivel de atención.

\section{CONCLUSIONES}

Del diagnóstico realizado en el presente artículo se observa capacidades institucionales deficientes de los GAM de menos de 5000 habitantes. Esta falencia tiene origen, entre otros, en variables como su escasa población, la falta de desarrollo de sus capacidades fiscales y la dificultad para ejecutar recursos. Esto pone en cuestión su habilidad para cumplir con las garantías, mandatos y competencias asignadas a este nivel de gobierno en la CPE, en áreas prioritarias para el desarrollo del Estado como son los sectores de salud y educación.

En este sentido, el Parágrafo III del Artículo 16 de la Ley No. 031 (Ley marco de autonomías y descentralización, 2010) establece como mandato de Estado la promoción de la fusión de aquellas UT con población inferior a cinco mil (5.000) habitantes (Ley marco de autonomías y descentralización, 2010). Como se vio, 74 de los GAM, el $22 \%$ de la UT existentes en la actualidad, se encuentran bajo este umbral. Estos GAM agrupan a el $1,65 \%$ de la población según los datos estimados a 2021 por el INE con base a datos del CNPV 2012, representando el 4,45\% de la población catalogada como pobre en el Estado Plurinacional de Bolivia y explican gran parte de la migración (inmigración y emigración) y el vaciamiento del área rural del país o su metropolización en cinturones de pobreza.

En consecuencia, se considera responsabilidad de Viceministerio de Autonomías y del SEA desarrollar mecanismos para la gestión supramunicipal o mancomunada de servicios en los GAM de menos de 5000 habitantes que así lo requieren con el objeto de garantizar un piso mínimo de servicios para todos los habitantes del
Estado Plurinacional de Bolivia especialmente en los sectores de salud y educación y realizar estudios detallados sobre sus proceso de inmigración y emigración conjuntamente con el INE, que permitan gestionar la información necesaria para realizar los procesos de reorganización territorial de forma informada y valorar el efecto político de éstos.

\section{REFERENCIAS}

Ameller, V. (1999). Biblioteca Virtual de la Cooperación Alemana. Obtenido de La problemática de los gobiernos municipales de escasa población. Consideraciones socioeconómicas para un tratamiento diferenciado: https://www.bivica.org/file/view/ id/4488

Böhrt, C. (2011). Análisis comparativo de los sistemas competenciales, su financiamiento y funcionamiento, en Bolivia, Colombia, Ecuador y Perú. La Paz - Bolivia: GIZ

Constitución Política del Estado (2 de febrero de 1967). Obtenido de https://www.wipo.int/ edocs/lexdocs/laws/es/bo/bo025es.pdf

Constitución Política del Estado (7 de febrero de 2009). Obtenido de https://sea.gob.bo/digesto/ CompendioNormativo/01.pdf

Deuer, A. (2016). Capacidades institucionales de las entidades territoriales autónomas en Bolivia. Repositorio Institucional Universidad Mayor de San Andrés. Tesis. Obtenido de: $\quad$ https://repositorio.umsa.bo/browse ?type=author\&value=Deuer+ Cenzano\%2C $+\%$ C3\%81lvaro+l

Deuer, A. \& Aparicio, V. (2018). Amalgamación (o fusión) de Gobiernos Locales en Bolivia. La Paz: UCB Tesis de maestría

Directrices y Clasificadores Presupuestarios 2017 (24 de junio de 2016). Obtenido de https://repositorio.economiayfinanzas.gob. bo/documentos/2018/VPCF/Documentos/ Clasificadores\%20Presupuestarios_2017.pdf

Directrices y Clasificadores Presupuestarios 2021 (2 de Julio de 2020). Obtenido de https://repositorio.economiayfinanzas. 
gob.bo/documentos/VPCF/DGPGP/2021/ Clasificadores_Presupuestarios_2021.pdf

Finot, I. (abril de 2016). Democratizar el Estado: A 25 años de una propuesta de descentralización para Bolivia. Obtenido de Página Institucional Fundación Friedrich Ebert: http://library.fes.de/ pdf-files/bueros/bolivien/12555.pdf

Hellsing, E. (2012). Municipal amalgamation Theory, Methodology and International Experiences A Desk Study for the Tuselog Programme. Obtenido de http://projects. sklinternational.se/tuselog/files/2013/03/ Municipal-consolidation_FINAL_LAYOUT.pdf

Instituto Nacional de Estadística. (2021). Página WEB Institucional. Obtenido de http://ine.gob. bo/

Ley de delimitación de Unidades Territoriales, Ley No. 339 (31 de enero de 2013). Obtenido de https://bolivia.infoleyes.com/norma/4315/ ley-de-delimitaci\%C3\%B3n-de-unidadesterritoriales-339

Ley de la Educación Avelino Siñani - Elizardo Pérez, Ley No. 070 (20 de diciembre de 2010). Obtenido de https://bolivia.infoleyes.com/ norma/2676/ley-de-la-educacion-avelinosi\%C3\%B1ani-elizardo-perez-070

Ley de Participación Popular, Ley No. 1551 (20 de abril de 1994). Obtenido de https:// bolivia.infoleyes.com/norma/639/ley-departicipaci\%C3\%B3n-popular-1551

Ley de Unidades Político Administrativas, Ley No. 2150 (20 de noviembre de 2000). Obtenido de https://bolivia.infoleyes.com/norma/4316/ ley-de-unidades-pol\%C3\%ADticoadministrativas-2150

Ley marco de autonomías y descentralización, Ley No. 031 (19 de julio de 2010). Obtenido de https://www.sea.gob.bo/ley-nro-31-ley-marcode-autonomias-y-descentralizacion-andresibanez/

Ley Orgánica de Municipalidades. (1985). Ley No. 696 (10 de enero de 1985). Obtenido de https:// docs.bolivia.justia.com/nacionales/leyes/ley696-1982-1985.pdf

Ministerio de Economía y Finanzas Públicas. (2021). Página WEB Institucional. Obtenido de https://www.economiayfinanzas.gob.bo/

Molina, F. (1997). Historia de la Participación Popular. La Paz - Bolivia: Ministerio de Desarrollo Humano de Bolivia

Normas Básicas del Sistema de Crédito Público, RS No. 218041 (29 de julio de 1997). Obtenido de https://bolivia.infoleyes.com/norma/783/ resoluci\%C3\%B3n-suprema-218041

Reforma parcial de la CPE de 1967, Ley N ${ }^{\circ} 1585$ (12 de agosto de 1994). Obtenido de https://www. lexivox.org/norms/BO-L-1585.html

Reglamento de la Ley $\mathrm{N}^{\circ} 339$, de 31 de enero de 2013, de Delimitación de Unidades Territoriales, DS No. 1560 (18 de abril de 2013). Obtenido de https://bolivia.infoleyes.com/ norma/4400/decreto-supremo-1560

Revollo, J. (2011). Líneamientos de un Pacto Fiscal. Problemas y Opciones de Política. La Paz: Fundación Konrad Adenaeur

Servicio Estatal de Autonomías. (2021). Página WEB Institucional. Obtenido de https://www. sea.gob.bo/

Tavares, A. (2018). Municipal Amalgamations and their effects: a literature review. Miscellanea Geographica. Regional Studies on Development Vol 22(1), 1 - 11 